\title{
Case Series of Seronegative Dengue Hemorrhagic Fever
}

\author{
Maheswaran Umakanth \\ Faculty of Health Care Sciences, Eastern University, Batticaloa, Sri Lanka \\ Email: mumakanth1972@gmail.com
}

How to cite this paper: Umakanth, M. (2018) Case Series of Seronegative Dengue Hemorrhagic Fever. Open Access Library Journal, 5: e4805.

https://doi.org/10.4236/oalib.1104805

Received: July 30, 2018

Accepted: August 21, 2018

Published: August 24, 2018

Copyright $\odot 2018$ by author and Open Access Library Inc.

This work is licensed under the Creative Commons Attribution International License (CC BY 4.0).

http://creativecommons.org/licenses/by/4.0/

\begin{abstract}
Dengue viral fever is an acute febrile illness which is transmitted by Aedes mosquitoes. It presented with body aches, rash, nausea, fever, headache, pain behind the eyes, and vomiting which are common symptoms of dengue. Dengue infections can result in a wide spectrum of disease severity ranging from an influenza-like illness such as dengue fever (DF) to the life-threatening dengue hemorrhagic fever (DHF) and dengue shock syndrome (DSS). During the dengue epidemic, we observed number of seronegative DHF. The descriptive retrospective study was conducted in the medical unit at Batticaloa, Teaching Hospital Sri Lanka. We collected data from seronegative dengue patient's medical records. The leakage was confirmed by periodic abdominal ultrasound and chest X-ray. The data were entered in the validated questionnaire. A total 11 seronegative DHF patients were participated in this study. Out of that 8 were male and 3 were female. Majority of patients were in 22 34 age group. The mean age was 26.36 years old. Interestingly, almost all seronegative DHF patients' lowest platelets were less than $28 \times 10^{9} / \mathrm{L}$. The minimum platelets count noted was $8 \times 10^{9}$. Raised liver enzymes were noted in almost all the cases. Even though lowest platelets were reported none of the patients needed platelets or blood transfusion. None of the patients end up with DSS.
\end{abstract}

\section{Subject Areas}

Infectious Diseases

\section{Keywords}

Seronegative Dengue Fever, Seronegative Dengue Hemorrhagic Fever

\section{Introduction}

Dengue is a viral infection transmitted by Aedes mosquitoes. Dengue is a flavi- 
virus with four different antigenically distinct serotypes (DENV1-4) [1]. The spread of dengue is thought to be due to a combination of factors such as, increased urbanization, population growth, migration and international travel and the difficulties of effective vector control. It is estimated that there are between 50 and 100 million cases of dengue each year, of which 500,000 are severe life-threatening infections [2]. The pathogenesis of severe disease is thought to be in part immune mediated, but the exact mechanisms remain unknown. Dengue infections can result in a wide spectrum of disease severity ranging from an influenza-like illness such as dengue fever (DF) to the life-threatening dengue hemorrhagic fever (DHF) and dengue shock syndrome (DSS). The febrile phase is characterized by continuing high fever lasting for 2 - 7 days [3]. Other features seen in the febrile phase include facial flushing/diffuse blanching erythema of the skin, myalgia, arthralgia, headache, nausea and vomiting. DHF is characterized by the acute onset of high fever and is associated with signs and symptoms similar to DF in the early febrile phase. Plasma leakage is the hallmark of DHF which occurs soon after the end of the febrile phase [4]. The characteristic laboratory finding for the disease is leukopenia. Other laboratory findings include thrombocytopenia, elevations of serum aminotransferase concentrations. Recognition of dengue specific IgM/IgG antibodies remains the most commonly used diagnostic tests. Usually, seroconversion occurs around 3 to 7 days following contact and, hence, testing of acute and convalescent sera may be necessary to make the diagnosis [5]. However, identification of early dengue infection may be made by detection of the dengue NS1 antigen. NS1 antigenemia is detectable within 24 hours of infection and up to 9 days following symptom onset [5].

The diagnosis of dengue fever is based on isolation of virus, viral RNA detection, or discovery of dengue-specific IgM, and IgG serum antibodies. There are pros and cons all these investigations. Isolation of dengue virus needs prolonged culture, which is expensive. Virus isolation technique not available in many parts of the Sri Lanka, moreover it is poor sensitivity. Doing antibody (IgM and IgG) another method to detect primary or secondary dengue infection, however antigenic cross-reactivity is the major setback [6]. However, detection of antibody test is widely used in Sri Lanka. Detecting NS1 antigen can give results in few hours or minute. Plasma leakage can be identified by abdominal ultrasonography or chest X-ray. Here I highlighted clinical and laboratory profile of eleven seronegative DHF [7].

\section{Methodology}

The descriptive retrospective study was conducted in the medical unit at Batticaloa, Teaching Hospital Sri Lanka over a period of 6 months from $15^{\text {th }}$ of March 2017 to $15^{\text {th }}$ of September 2017. According to the epidemiology unit, ministry of health Sri Lanka stated reported that it was the bad period in Sri Lanka, where highest number of dengue cases were reported Island wide. We collected data from patient's medical records. The data were entered in the validated questionnaire. During this period, we don't have the facility to do dengue antigen test. 
We used to do dengue IgM antibody after $5^{\text {th }}$ day of the fever, however in case of negative IgM antibody test, we arranged repeat test 2 - 3 days later. If both tests were negative then only, we labeled as seronegative. All patients above 12 years with confirmed dengue hemorrhagic fever with negative dengue-IgM antibodies were included in this study. The leakage was confirmed by periodic abdominal ultrasound and chest X-ray. If small amount of pericolic free fluid without progressive changes were excluded from this study. The patients with concomitant malaria, typhoid, and leptospirosis were excluded from the study. Laboratory investigations such as hemoglobin, total and differential leucocyte counts, platelet counts, hematocrit, liver function tests, blood urea and serum creatinine were extracted from the patients' medical records. Patients clinical presentations were harvested from the patient's medical records. If any uncertainty about investigations or diagnosis, we excluded from the study. Written consent was taken from all the participants and approval was taken from the head of the institution for conducting this study.

\section{Results}

A total 11 seronegative DHF patients were willing to participate in this study. Out of that 8 were male and 3 were female. Maximum patients were in $22-34$ age group. The mean age was 26.36 years-old. The platelets count at presentation was less than $80 \times 10^{9} / \mathrm{L}$ in around $8(72.7 \%)$ of cases. However, it kept on falling further during hospitalization. Interestingly, almost all seronegative DHF patient's lowest platelets were less than $28 \times 10^{9} / \mathrm{L}$. The minimum platelets count noted were $8 \times 10^{9} /$ Raised liver enzymes were noted almost all the cases. Even though lowest platelets were reported none of the patients needed platelets or blood transfusion. None of the patients end up with DSS. When we consider clinical profile fever (100\%) was the universal followed by headache was [8] $81.81 \%$, myalgia $8(72.72 \%)$, and skin rash $36.3 \%$. All patient's renal functions were normal throughout the hospital stay. Usually we full blood count three times a day throughout the period haemoglobin and haematocrit were slightly fluctuated but within the normal range (Table 1).

\section{Discussion}

We collected all data during the dengue epidemic period in east part of the Sri Lanka. Even though clinical findings suggestive with evidence of plasma leakage favor DHF, however negative IgM-dengue antibody make diagnostic challenge for us. Finally, 11 seronegative DHF were analyzed for this study. A Primary dengue infection is characterized by a slow and low level of dengue specific IgM antibody which is apparent in 3 - 5 days after the onset of fever. In addition to that low level of IgG-dengue antibody was observed. However, in secondary infection dengue specific IgG antibody increase extremely rapidly to reach high level at days 1 to 2 after onset of symptoms [9]. Interestingly, dengue specific IgM antibody level are significantly lower in secondary dengue infections. Furthermore, some anti-dengue IgM false-negative reactions are observed during 
Table 1. Sex, age and laboratory profile of seronegative dengue hemorrhagic fever.

\begin{tabular}{|c|c|c|c|c|c|c|c|c|c|c|c|}
\hline Case & 1 & 2 & 3 & 4 & 5 & 6 & 7 & 8 & 9 & 10 & 11 \\
\hline Sex & M & M & M & $\mathrm{F}$ & M & $\mathrm{F}$ & M & M & $\mathrm{F}$ & M & M \\
\hline Age & 23 & 25 & 18 & 24 & 34 & 32 & 18 & 26 & 31 & 18 & 23 \\
\hline NS1 antigen & - & - & - & - & - & - & - & - & - & - & - \\
\hline IgM-dengue antibody & - & - & - & - & - & - & - & - & - & - & - \\
\hline IgG-dengue antibody & + & + & + & + & + & + & + & + & + & + & + \\
\hline $\begin{array}{l}\text { Platelets at admission } \\
\left((150-400) \times 10^{9} / \mathrm{L}\right)\end{array}$ & 105 & 78 & 75 & 107 & 55 & 45 & 67 & 100 & 65 & 64 & 64 \\
\hline Lowest platelets $\times 10^{9} / \mathrm{L}$ & 9 & 12 & 28 & 23 & 24 & 8 & 11 & 15 & 12 & 18 & 17 \\
\hline Platelets transfusion & - & - & - & - & - & - & - & - & - & - & - \\
\hline Blood transfusion & - & - & - & - & - & - & - & - & - & - & - \\
\hline AST (5 - $30 \mathrm{IU} / \mathrm{L})$ & 78 & 55 & 124 & 202 & 58 & 121 & 98 & 96 & 68 & 76 & 145 \\
\hline $\operatorname{ALT}(5-30 \mathrm{IU} / \mathrm{L})$ & 55 & 48 & 58 & 95 & 45 & 76 & 78 & 70 & 55 & 48 & 68 \\
\hline \multicolumn{12}{|l|}{ Blood urea $(8-25 \mathrm{mg} / \mathrm{dl})$} \\
\hline $\begin{array}{l}\text { Serum creatinine } \\
(0.8-1.3 \mathrm{mg} / \mathrm{dl})\end{array}$ & 0.9 & 1.1 & 0.8 & 0.9 & 1.0 & 1.1 & 0.8 & 0.9 & 1.2 & 1.0 & 0.9 \\
\hline Number of days in hospital & 6 & 6 & 4 & 5 & 5 & 6 & 7 & 5 & 5 & 6 & 4 \\
\hline Complication & No & No & No & No & No & No & No & No & No & No & No \\
\hline
\end{tabular}

secondary infections. The Pan American Health Organization (PAHO) guidelines revealed that $80 \%$ of all dengue cases have noticeable IgM antibody by day five of illness, and $93 \%$ - $99 \%$ of cases have noticeable IgM by day six to ten of illness, which may then remain detectable for over 90 days [8].

Antibody response to dengue virus depends to the host immune status. When, previously been not infected with a flavivirus or immunized with flavivirus vaccine such as yellow fever, Japanese encephalitis and tick-bone encephalitis had dengue fever causes slow and steady increase of IgM antibody [10]. These dengue specific IgM antibodies are detectable in $50 \%$ of patients by day $3-5$ after the onset of illness, it increasing to $80 \%$ by day of $5 \%$ and $99 \%$ by day of ten. IgM levels peak about two weeks after the onset of symptoms and then decline generally to undetectable levels over 2 - 3 months [11]. In case of secondary dengue infection, antibody titres raise speedily and react generally against many flaviviruses. The main immunoglobulin isotype is IgG which is obvious at high levels, even in the acute phase, and persists for periods lasting from 10 months to life [12]. Negative dengue specific IgM antibody cannot rule out dengue fever. A meta-analysis has conducted by Blacksell et al., which clearly mentioned that the sensitivity and specificity of acute dengue fever which less than 7 days were $73 \%$ and $88 \%$, however this pattern changed markedly in late febrile (7 - 10 days) sample were $96 \%$ and $90 \%$, respectively [13].

In this study almost all the patient had positive dengue IgG antibody, which indicates that all eleven-patients had secondary dengue infection, this may be the 
reason for negative IgM antibody response, which may take time to develop. All most all seronegative dengue patients had the evidence of liver involvement. The spectrum of liver involvement ranges from asymptomatic elevation of liver enzymes, fulminant hepatic failure, tender hepatomegaly, and jaundice. The elevation of AST enzyme is normally greater than the elevation in the level of ALT in the dengue patients.

Hospital admission criteria for DF is difficult to define, it depends on the patient's clinical status. However, most of the time patients come with low platelets. In this case series majority of the patients had less than $100 \times 10^{9 /} / \mathrm{L}$ of platelets on admission. However, it kept on falling further during hospitalization. Furthermore, the DHF patients with secondary infections have a more severe clinical picture than the patients with primary infections. However, the difference in AST and ALT levels in primary and secondary infections was statistically not significant [14] [15]. In this case series average hospital stay was 5.36 days, furthermore number of hospital stay not depends on platelets drop. Even though platelets were dropped below $20 \times 10^{9 /} / \mathrm{L}$ in many cases, but patients were not received any blood or blood products. The clinical presentations and laboratory findings are similar in both seropositive and seronegative DHF.

\section{Conclusion}

Dengue IgM serology has low sensitivity during the early phase of dengue fever. Negative IgM cannot rule out the dengue fever. During the dengue epidemic, even though negative dengue specific IgM antibody, if clinical finding suggestive of leakage, first we should treat like DHF rather than wasting the time to find the dengue serology.

\section{Limitations of the Study}

There are number of limitations of this study, firstly total number of seronegative DHF is only eleven, which is fairly small amount. We didn't do NS1 antigen test, because of non-availability. We didn't compare this group with seropositive groups.

\section{Conflict of Interest}

None declared by the author.

\section{Availability of Data and Material}

All data gathered during this study are included in this published article.

\section{Approval for Conducting Research}

Approval was taken from the head of the institution.

\section{Funding}

This research received no funding support. 


\section{References}

[1] WHO (2017) Dengue and Severe Dengue. World Heal Organ.

[2] WHO (2009) Dengue: Guidelines for Diagnosis, Treatment, Prevention and Control. World Heal Organ.

[3] Ministry of Health Sri Lanka (2012) Guidelines on Management of Dengue Fever \& Dengue Haemorrhagic Fever in Adults [Internet]. 1-39.

http://www.epid.gov.lk/web/images/pdf/Publication/guidelines_for_the_manageme nt_of_df_and_dhf_in_adults.pdf

[4] World Health Organization (2009) Dengue: Guidelines for Diagnosis, Treatment, Prevention, and Control. Special Programme for Research and Training in Tropical Diseases [Internet], 147.

http://whqlibdoc.who.int/publications/2009/9789241547871_eng.pdf

[5] Rigau-Perez, J.G., Clark, G.G., Gubler, D.J., et al. (1998) Dengue and Dengue Haemorrhagic Fever. Lancet, 352, 971-977. https://doi.org/10.1016/S0140-6736(97)12483-7

[6] Teng, C.L. and Wong, C.H. (2013) Does Negative IgM Dengue Serology Rule out Dengue Fever in an Adult with Fever for Three Days? Malaysian Family Physician, 8, 26-27.

[7] Johnson, B.W., Russell, B.J., Lanciotti, R.S. and Icrobiol, J.C.L.I.N.M. (2005) Serotype-Specific Detection of Dengue Viruses in a Fourplex Real-Time Reverse Transcriptase PCR Assay. Journal of Clinical Microbiology, 43, 4977-4983.

[8] Guzman, M.G. and Kouri, G. (2004) Dengue Diagnosis, Advances and Challenges. International Journal of Infectious Diseases, 8, 69-80.

https://doi.org/10.1016/j.ijid.2003.03.003

[9] Ruiz, D., Vázquez, S., Villegas, E., Balmaseda, A., Pupo, M., Alvarez, M., et al. (2017) Virological and Serological Markers in Dengue Patients from Venezuela and Nicaragua. OALib [Internet], 4, 1-16. http://www.oalib.com/paper/pdf/5280576

[10] Vazquez, S., Perez, A.B., Ruiz, D., Rodriguez, R., Pupo, M., Calzada, N., Gonzalez, L., Gonzalez, D., Castro, O., Serrano, T. and Guzman, M.G. (2005) Serological Markers during Dengue 3 Primary and Secondary Infections. Journal of Clinical Virology, 33, 132-137.

[11] Innis, B., et al. (1989) An Enzyme-Linked Immunosorbent Assay to Characterize Dengue Infections Where Dengue and Japanese Encephalitis Co-Circulate. The American Journal of Tropical Medicine and Hygiene, 40, 418-427. https://doi.org/10.4269/ajtmh.1989.40.418

[12] Kuno, G. and Gomez, I.G.D. (1991) An ELISA Procedure for the Diagnosis of Dengue Infections. Journal of Virological Methods, 33, 101-113. https://doi.org/10.1016/0166-0934(91)90011-N

[13] Guzman, M.G., Halstead, S.B., Artsob, H., Buchy, P., Farrar, J., Gubler, D.J.H.E., Kroeger, A., Margolis, H.S., Martinez, E., Nathan, M.B., Pelegrino, J.L., Simmons, C., Yoksan, S. and Peeling, R. (2010) Dengue: A Continuing Global Threat. Nature Reviews. Microbiology, 8. https://doi.org/10.1038/nrmicro2460

[14] Jayanta, S. and Vishal, S. (2015) Dengue and Its Effects on Liver. World Journal of Clinical Cases [Internet], 3, 125-131.

http://www.ncbi.nlm.nih.gov/pubmed/25685758\%5Cnhttp://www.pubmedcentral.n ih.gov/articlerender.fcgi?artid=PMC4317605

https://doi.org/10.12998/wjcc.v3.i2.125 
[15] Vinodh, B.N., Bammigatti, C., Kumar, A. and Mittal, V. (2005) Dengue Fever with Acute Liver Failure. Journal of Postgraduate Medicine [Internet], 51, 322-323. http://www.ncbi.nlm.nih.gov/pubmed/16388178 